Voix et Images

\title{
Le tremblement de la conscience. Entretien avec Louky Bersianik
}

\section{Louise Dupré}

Volume 17, numéro 1 (49), automne 1991

Louky Bersianik

URI : https://id.erudit.org/iderudit/200939ar

DOI : https://doi.org/10.7202/200939ar

Aller au sommaire du numéro

Éditeur(s)

Université du Québec à Montréal

ISSN

0318-9201 (imprimé)

1705-933X (numérique)

Découvrir la revue

Citer ce document

Dupré, L. (1991). Le tremblement de la conscience. Entretien avec Louky

Bersianik. Voix et Images, 17(1), 11-21. https://doi.org/10.7202/200939ar d'utilisation que vous pouvez consulter en ligne.

https://apropos.erudit.org/fr/usagers/politique-dutilisation/ 


\section{Le tremblement de la conscience Entretien avec Louky Bersianik}

par Louise Dupré, Úniversité du Québec à Montréal

V. \& I.: Louky Bersianik, où êtes-vous née?

L. B.: Je suis née à Montréal, rue Saint-Denis, puis nous sommes déménagés sur la rue de Lanaudière, entre Rachel et Marie-Anne. Je suis donc née en plein cœur de Montréal: Mon père était professeur de français à l'école normale Jacques-Cartier de Montréal: c'était la seule école normale au Québec où les professeurs étaient nommés en vertu d'une charte gouvernementale. La situation était dotée d'un certain prestige, mais était mal payée comme l'était tout le secteur de l'enseignement à l'époque. Il y avait beaucoup de livres à la maison. J'ai vécu dans un milieu intellectuel, au sens où les idées et l'instruction comptaient énormément.

V. \& I.: Vous dédiez la Main tranchante du symbole à la mémoire de votre mère qui sacrifía ses rêves pour'mettre au monde onze enfants. Est-ce une prise de conscience d'adulte ou si, enfant, vous perceviez déjà ce sacrifice de votre mère?

L. B.: Enfant, je percevais la douleur, la souffrance de ma mère. J'étais révoltée contre cette souffrance-là. À l'époque, la grossesse était perçue comme une maladie: ma mère était donc constamment malade, car en plus d'être enceinte, elle était de santé fragile. Elle était musicienne, c'était une très bonne musicienne et elle aurait voulu devenir pianiste de concert. Elle était très résolue pour les dix enfants qui ont survécu, surtout pour ses filles, parce qu'elle avait été obligée de sacrifier ses rêves. Elle nous a donc mises au couvent pour qu'on puisse avoir une éducation décente.

V. \& I.: Les auteurs affirment souvent qu'ils ont été marqués par des livres de leur enfance. Et vous?

L. B.: Dans mon enfance, je ne pourrais pas dire que ce sont des livres qui m'ont marquée, mais l'obligation d'écrire tous les dimanches une lettre à mes parents quand j'étais au couvent. J'ai pris là la gestuelle de l'écriture. Tous les dimanches, alors que les autres enfants allaient au parloir, j'écrivais une lettre rassurante à mes parents pour leur dire que tout allait bien, même s'il y avait eu des 
drames dans le courant de la semaine. J'ai su très tôt que je serais traversée par l'écriture, que ma raison de vivre serait là.

V. \& I.: Vous avez fait votre cours classique, je crois...

L. B.: Ma mère est allée solliciter une bourse au Collège Jésus-Marie. J'ai pu faire mes études classiques dans un collège de riches. J'étais une petite fille pauvre dans un milieu riche: c'était très dur, mais je me suis tout de même fait de bonnes amies. Ensuite, je suis allée à l'Université de Montréal, en lettres françaises. J'ai fait ma maitrise avec le père Ernest Gagnon. Il a été un réveilleur de conscience, je le considérais comme mon maitre. Il a permis que je fasse, comme mémoire, une introduction à une vie de Jésus que Bernanos n'avait jamais écrite, mais qu'il voulait écrire. Lui seul pouvait accepter un sujet si près de la fiction. Plus tard, à Paris, j’ai rencontré Albert Béguin, puis le fils de Bernanos. Albert Béguin voulait absolument faire publier mon mémoire, mais il aurait fallu que je le retravaille dans ce sens-là et je n'avais plus le goût de revenir là-dessus. J'étais incroyante.

V. \& I.: Au moment où vous avez fait votre mémoire sur Bernanos, vous étiez croyante?

L. B.: Très tôt, -j'ai commencé à aller à la messe au restaurant, comme on disait. Je n'étais pas vraiment croyante dans la mesure où je doutais beaucoup. Cela correspondait chez, moi à l'éveil de la sexualité. À l'époque, il était très difficile de concilier religion et sexualité. Puis j'ai posé beaucoup de questions à la philosophie: on n'étudiait pas la philosophie, on étudiait saint Thomas d'Aquin. Quand j'ai entrepris mon mémoire, je crois que j'ai voulu retrouver mes racines religieuses parce que mes parents étaient très religieux. Et, bizarrement, ce travail m'a éloignée de la croyance religieuse, il m'a permis de m'en défaire.

V. \& I.: Vous avez aussi commencé un doctorat ...

L. B.: J'ai fait ma scolarité de $\mathrm{Ph} . \mathrm{D}$. à Montréal et réussi mes examens. Puis, grâce à une bourse du gouvernement français, j’ai pu aller étudier à la Sorbonne. Je n'ai pas terminé le doctorat. Je voulais faire une thèse sur Apollinaire, Henri Michaud, Saint-John Perse et Paul Éluard. Le projet avait pour titre "Poésie nécessaire", mais on m'a dit qu'il s'agissait davantage d'un sujet de livre que d'un sujet de thèse: il fallait travailler sur des écrivains qui étaient décédés depuis plus de cinquante ans. Mais à Paris, j'ai écrit passablement, j'ai donné des textes à la radio. En revenant à Montréal, j'ai rencontré Jean Letarte, qui allait devenir mon mári. Nous avons travaillé beaucoup ensemble, nous avons fait du cinéma, de la chanson, j'écrivais les textes de ses films.

V. \& I.: Il me semble que vous avez aussi travaillé à Radio-Canada... 
L. B.: Jean et moi, nous avons fait ensemble une émission radiophonique pour les jeunes qui s'appelait la Boîte aux merveilles. Mon mari écrivait les chansons et nous faisions les contes: on se racontait les histoires et j'écrivais le texte. On en a fait 45 . Puis, pendant sept ans, j’ai écrit pour la télévision une autre émission pour enfants, Chez Hélène. C'est ce que j'appelle de l'alimentaire, mais c'était intéressant. J'écrivais des textes pour la radio, pour la télévision. J'ai aussi fait des dialogues et des scénarios de films.

\section{V. \& I.: Votre premier ouvrage publié est-il l'Euguélionne?}

L. B.: Non, quatre contes pour enfants ont été publiés au Centre de psychologie et de pédagogie de Montréal, avec des illustrations de Jean Letarte. Ils sont maintenant épuisés. Un des contes a remporté le Prix de la province.

\section{V. \& I.: Considérez-vous l'Euguélionne comme un roman?}

L. B.: Oui, dans la mesure où l'Euguélionne, comme personnage de fiction, assume le second niveau de narration. Mais l'Euguélionne est construit en trois volets: chaque volet a un ton, chacun peut avoir son genre. À l'époque, souvenons-nous, la notion de genre littéraire était remise en question. Je n'avais pas écrit « roman " sur la couverture, je voulais inscrire seulement triptyque. Mais on ne me l'a pas permis pour des raisons commerciales.

\section{V. \& I.: Et le Pique-nique sur l'Acropole?}

L. B.: Comment appeler ce livre? Je ne sais pas ... Il s'agit d'un texte centré sur la philosophie et la psychanalyse. Mais il y a aussi une fiction, des personnages comme dans l'Euguélionne. La narratrice, c'est Ancyl, ce n'est pas moi. On retrouve donc ici aussi une construction romanesque. Chose sûre, ces deux livres appartiennent à ce qu'on pourrait appeler de la théorie-fiction. Mais mes prochains romans seront de "vrais" romans. Pourtant, ce n'est pas une concession, même si je me suis fait beaucoup taper sur les doigts parce que j'écrivais des textes "impurs".

V. \& I.: Vous avez écrit, dans la Main tranchante du symbole, qu'on fait de la poésie non pas avec des idées mais avec des mots, selon le principe mallarméen. Avez-vous une posture d'écriture différente selon le genre que vous pratiquez?

L. B.: Absolument. Il y a vraiment une différence entre l'approche de la fiction en prose et de la poésie. La poésie est comme une forme en mouvement. C'est un moment du moúvement qui est saisi soit en accéléré, soit dans un moment privilégié. La poésie va chercher le mouvement intérieur d'un objet qui rend caduques nos perceptions antérieures. La prose, quant à elle, nous offre le déroulé du mouvement, elle le montre au ralenti. La poésie est une quête de l'univers, soit de l'invisible, soit de ce qui est trop visible. Certains faits sont 
tellement évidents que personne ne les voit. Mais il y a des liens entre la poésie et la fiction en prose. Dans mes ateliers d'écriture, je précise qu'il faut expliciter ce qui est trop implicite et impliciter ce qui est trop explicite. Je ne veux pas écrire des textes hermétiques. $\mathrm{Ni}$ tout donner. L'Euguélionne semble un texte explicite lors d'une première lecture, mais il y a des réseaux implicites.

V. \& I.: Comment travaillez-vous la poésie?

L. B.: J'ai toujours voulu pratiquer une poésie pure. Pour moi, la poésie est comme un jeu de hasard qui devient une absolue nécessité. Cela débute par une fête du langage, par une errance, par le ludique: jeux de mots, lapsus, dérives', disséminations. Je travaille le signifiant. Quand je donne des ateliers d'écriture, je dis: Laissez vos peines d'amour et vos colères à la porte. Si on part avec des idées, on ne créera pas d'idées, on va reproduire du sens. Chez moi, la pratique rejoint la théorie. Le langage est une expression en soi. Il s'agit d'une exploration qui conduit à l'expression de soi. On commence donc avec l'expression en soi pour arriver à l'expression de soi. Je travaille beaucoup la translinéarité: c'est le visible du texte, alors que la linéarité est le lisible du texte.

V. \& I.: C'est ce que vous vouliez signifier quand vous affirmez que la dynamique du rapport langue/ecriture doit se poser en termes d'exploration et de transformation du langage?

L. B.: J'ai beaucoup étudié les théories du texte et beaucoup pratiqué la technique des générateurs de Ricardou que j’ai développée dans mes ateliers et adaptée à mes besoins. Pour moi, la contrainte consciente permet plus de liberté que celle qu'on retrouve dans l'écriture automatique ou dans l'écriture dite «inspirée ", là où sont véhiculées énormément de contraintes inconscientes, ne serait-ce que les règles de grammaire ou de stylistique. Ce n'est pas le vouloir-dire, mais le vouloir-écrire qui prédomine chez moi. Et, à travers les contraintes, j'arrive à ma thématique personnelle, à mon univers. Quand j'arrive à un thème sans le vouloir, je trouve la joie, l'étonnement et l'émerveillement de l'écriture, je suis allée trouver quelque chose de très profond en moi.

Je donne un exemple. Si on m'avait commandé un texte sur le thème des sœurs ennemies, j’aurais sans doute écrit un texte prévisible. Le magazine la Vie en rose nous avait demandé d'écrire un texte dans lequel il y aurait la phrase suivante: Une fourmi flottait sur sa margarita. En partant du signifiant, c'est-à-dire de la graphie, des sonorités, je suis arrivée au thème des sœurs ennemies. Mon texte s'intitule "Sosie". La même chose s'est produite avec le premier poème de Axes et eau, * Lacrimacorpus dissolvens ", où, à partir de deux parenthèses vides qui accompagnent le texte depuis le début, je suis arrivée à découvrir, en terminant le poème, ce que contenaient 
ces parenthèses, c'est-à-dire le thème fondamental du texte. Les contraintes nous donnent des pistes d'écriture qui sortent de l'ordinaire.

\section{V. \& I.: Et dans les essais?}

L. B.: J'écris toujours entre la dictée de l'inconscient et le tremblement de la conscience. Dans la fiction et dans la poésie, je reste plus près de l'inconscient, alors que dans l'essai, je me retrouve nécessairement plus collée au tremblement de la conscience. Mais les deux jouent constamment l'un par rapport à l'autre et l'inconscient demeure présent dans n'importe lequel texte, même les essais. Dans le Pique-nique sur l'Acropole, où il y a sept femmes, je me suis rendu compte après coup qu'on était sept filles dans ma famille.

V. \& I.: Travaillez-vous avec des contraintes uniquement dans la poésie?

L. B.: Non. Dans le Pique-nique sur l'Acropole, pour revenir à ce titre, j'ai commencé par une contrainte et je l'ai laissée ensuite parce qu'elle m'avait menée à ma thématique. La contrainte offre des pistes d'écriture, mais je n'utilise pas la contrainte pour la contrainte: à un moment donné, il faut la quitter, sauf dans des textes courts comme "Sosie".

V. \& I.: Dans votre texte * Là lanterne d'Aristote *, dans la Théorie, un dimanche, vous dites que vous avez travaillé le premier poème de Axes et eaux à partir de la figure de la toile d'araignée. S’agit-il aussi d'une contrainte?

L. B.: La toile d'araignée n'est pas une contrainte textuelle, mais une forme qui s'inscrit dans le texte, donc quelque chose qui m'a beaucoup énergisée. Si on observe la toile de l'araignée à partir d'un de ses rayons, on s'aperçoit qu'on voit toujours la spire précédente et la spire qui s'en vient. On voit, en même temps, le passé et l'avenir du texte. Je reprends des points qui ont été amorcés dans le texte précédent et, ensuite, je projette ce qui va s'écrire dans le suivant. C'est mon regard sur la toile. Il y a une continuité d'une spire à l'autre, une continuité dans le temps de l'écriture. Et la présence de cette forme transforme constamment mon texte qui devient la maison de l'araignée. Mon texte est une maison.

V. \& I.: On vous considère comme une auteure de la modernité et, d'ailleurs, vous êtes consciente de votre investissement dans ce courant au Québec. Finalement, vous êtes beaucoup plus proche de la génération qui vous suit que de celle de l'Hexagone par votre pensée, par votre travail de déconstruction, par ce travail d'exploration de la langue. Vous avez d'ailleurs publié souvent dans la Nouvelle Barre du jour. D'où vous vient cette nécessité? 
L. B.: Si on considère mon âge, je devrais être en effet beaucoup plus près de la génération de l'Hexagone. Mais je n'ai pas été reconnue par cette génération. Je voulais publier à l'Hexagone, mais je n'ai pas eu la chance ... peut-être parce que j'étais une femme ou qu'on n'aimait pas ce que je faisais, je ne sais pas.

V. \& I.: Est-ce que vous y aviez déjà soumis des textes?

L. B.: Gaston Miron connaissait mes textes, certains avaient même été publiés dans des revues à Paris. Je les avais lus à la Maison canadienne. Je lui avais demandé de me publier. À cette époque-là, c'était dans les années 1950, en 1958 je crois, il m'avait dit qu'il m'aurait fallu attendre trois ans. Je ne voulais pas attendre aussi longtemps. Autrement dit, je ne faisais pas partie du groupe ...

V. \& I.: Peut-être que ce que vous faisiez à l'époque n'entrait pas dans la mentalité de l'Hexagone ... Votre vision de la poésie était sans doute déjà très moderṇe, très influencée par, l'apport de Mallarmé ...

L. B.: Oui. Quand j'ai commencé à étudier la théorie du texte, je me disais en effet que j'écrivais des poèmes qui n'étaient pas étrangers à cette vision. J'ai eu la chance de pouvoir publier dans la Nouvelle Barre du jour. Mais je n'ai jamais publié de petits recueils de poèmes, comme cette génération l'a fait. Les jeunes de la Nouvelle Barre du jour et des Herbes rouges faisaient une recherche poétique de 10,20,30 ou 50 pages et ils la publiaient. Javais l'esprit de cette génération-là, mais cette façon de préparer une publication ne correspondait pas à ma manière.

V. \& I.: Il faut dire que les auteurs de votre génération publiaient beaucoup moins qu'on ne publie aujourd'hui ...

L. B.: On attendait... Miron n'a pas publié beaucoup non plus ...

V. \& I.: Même si vous vous sentiez près de la génération de la Nouvelle Barre du jour, vous vous êtes tout de même montrée critique par rapport à leur travail, au colloque * Vouloir la fiction, la modernité " en 1984. Quels étaient pour vous les points négatifs de ce courant?

L. B.: Il y a des textes qui m'ont ravie, qui m'ont transportée. Cela dit, je trouvais que la modernité n'était pas sans produire de tics. Le thème de la quotidienneté, par exemple, devenait omniprésent dans les textes. Il y avait aussi du snobisme. Et du laisser-aller chez certains poètes: j'avais l'impression que certains publiaient leurs brouillons. Et puis on restait encore dans une symbolique patriarcale.

V. \& I.: Même chez les femmes?

L. B.: Beaucoup moins chez les femmes. C'est un fait très intéressant dans la modernité québécoise: il y a autant de femmes que d'hommes 
qui écrivent de la poésie. Et c'étaient surtout les femmes qui me considéraient comme faisant partie des leurs. Elles acceptaient que je puisse mélanger les genres.

V. \& I.: On vous sent parfois un peu amère...

L. B.: J'ai été isolée dans ma génération, avec toutes les conséquences qui en découlent. Par exemple, quand on parlait de nouvelle écriture, mon nom n'était pas mentionné. Quand on parlait de la génération de l'Hexagone, on insistait sur le pays. Et moi, je n'ai jamais accroché à la thématique nationaliste. On a permis aux poètes nationalistes de parler du pays, mais si j'aborde une thématique féministe, c'est de l'idéologie. Deux poids, deux mesures, je trouve. Dans l'Euguélionne, mon point de départ n'a pas été la théorie féministe, ce qui aurait abouti à un livre doctrinaire: c'est à partir du vécu que je suis remontée à certaines constatations théoriques. Quant à l'amertume, je sens que je suis en train de la liquider: séjourner à l'étranger relativise beaucoup de choses. Maintenant, j'accepte qu'on me dise que j'écris «à côté de la plaque». Forcément, puisque la plaque est patriarcale: pour être valide, le point de vue sur toute question doit être masculin.

V. \& I.: LEuguélionne serait de l'ordre de l'utopie plutôt que du roman à thèse?

L. B.: Oui, car il met en scène un personnage archétypal et donne la priorité à l'imaginaire. Il s'agit d'un personnage mythique, qui donne de l'énergie aux gens, qui lés porte en avant. Et cela passe par la déconstruction des valeurs culturelles. Il faut aussi déconstruire. Et je ne suis pas d'accord avec les féministes qui soutiennent que déconstruire l'imaginaire patriarcal, c'est le conforter. Je veux retourner aux sources du patriarcat. Réouvrir le procès d'Oreste. Voilà pourquoi je suis allée passer six mois en Grèce cette année. On n'eśt pas conscient que la réalité part des mythologies. Si on ne comprend pas cela, il y a beaucoup de choses qu'on ne saisit pas. Il faut absolument sortir des catégories mentales issues du patriarcat et dans lesquelles se meuvent tous nos raisonnements. On ne peut pas faire l'économie de cette critique des mythes patriarcaux pour pouvoir réaliser l'utopie de la gynilité.

V. \& I.: La mythologie, et plus particulièrement la mythologie grecque, est primordiale dans votre réflexion. D'où vous vient cet intérêt?

L. B.: C'est très ancien. Le grec, je l'ai appris bien avant d'aller au collège. Je l'ai appris de mon père et de ma mère: ma mère faisait répéter son grec à mon père. Quand j'ai entendu prononcer toutes ces lettres-là - alpha, bêta, kappa, delta, etc. - je me suis dit qu'un jour j'écrirais un roman où il y aurait 25 personnages, comme les 25 lettres grecques. Je n'ai jamais écrit ce roman, bien que dans l'Euguélionne, 
toutes les lettres grecques soient représentées dans. les noms des personnages. Par exemple, il y a Deltanu, Delta est nue car elle se fait avorter. In y a Alfred Oméga, c'est l'alpha et l'oméga; Omicronne la minuscule; Bétamu, le mari d'Exil, qui est bêta et qui mue.

V. \& I.: Vous étiez intéressée par le signifiant, encore une fois...

L. B.: J'ai éprouvé une fascination pour l'alphabet grec avant de la développer pour la Grèce et la mythologie. Je trouvais la mythologie fastidieuse, j’y suis revenue il y a quelques années. Pour moi, il s'agit davantage d'une conscience que d'une grille. Les effets de la mythologie sont encore présents. Quand je parle d'Oreste, c'est très actuel. Les hommes essaient encore de se réapproprier la maternité. La mythologie nous aide à construire une mémoire du passé pour ensuite penser le présent.

V. \& I.: Votre écriture se situe très loin de ce qu'on pourrait appeler une écriture autobiographique. Et pourtant, vous avez mentionné déjà que vous écrivez pour dénouer les drames de votre enfance ...

L. B.: Tant que je ne suis pas reconnue comme un être humain à part entière sur la planète, comment me faire reconnaître comme Québécoise, comme Louky Bersianik ou Lucile Durand? Quand j'écrivais l'Euguélionne, je me disais qu'il me fallait passer par là avant de me donner la-permission d'écrire des livres plus subjectifs. Mais il me semble que je doive continuer, parce qu'il y a encore des points à creuser. C'est jour après jour que cette reconnaissance-là nous est refusée.

Mais peut-être cela vient-il de mon enfance: il est difficile d'être reconnue dans une famille de dix enfants. Puis il y a sans doute ma timidité. Pourtant, je m'exprime beaucoup plus dans ma poésie. Jai lu dans une soirée de poésie "Corps de noce corps de divorce": il s'agit d'un texte très personnel.

V. \& I.: Mais même dans la poésie, vos personnages vous suivent. Vous écrivez rarement à la première personne, c'est-à-dire un je qui représente la personne linguistique de l'auteure...

L. B.: C'est très vrai. J'ai beaucoup de mal à écrire je. Pourquoi? Je ne saurais le dire. J'ai besoin de transposer ma vie à travers différents personnages. Cela reste pour moi une question en suspens. Peut-être parce que je est un autre et que j'ai le sentiment de ne jamais arriver au je. Je n'existe pas finalement. Il reste tellement mouvant. Ce n'est ni l'autobiographique ni l'anecdotique qui peuvent le définir. Si j'écrivais au je dans un livre, ce je ne me représenterait pas. L'autobiographique n'a aucun intérêt pour moi, je dois absolument transposer.

V. \& L.: N'y a-t-il pas un lien avec la psychanalyse? Vous êtes très critique par rapport à la psychanalyse... 
L. B.: J'ai d'ailleurs été six ans en analyse. Mais c'est comme si ce passage m'avait permis de me défaire de la psychanalyse, comme mon mémoire de maîtrise sur Bernanos m'avait permis de laisser la religion de côté. Je trouve formidable la cure psychanalytique quand on en a besoin. Mais je suis très critique par rapport à la doctrine psychanalytique: il s'agit vraiment d'une doctrine, au sens fort, parce qu'elle est construite sur des préjugés sexuels ancestraux.

V. \& I.: Considérez-vous qu'il y a un cërtain lyrisme dans votre écriture?

L. B.: D'une certaine façon, oui,-parce que l'écriture est traversée par une passion. À partir du moment où il y a de la passion, il y a nécessairement du lyrisme. Mais ce n'est pas ce qui prédomine.

V. \& I.: Diriez-vous que la posture épique vous convient mieux?

L. B.: Oui, très certainement. Je parlais tout à l'heure des trois volets de l'Euguélionne: je dirais que le premier volet est un volet épique; le deuxième, un volet onirique et le troisième, un volet discursif. La posture épique me semble primordiale parce qu'on a à construire une épopée de femmes, ce qu'on n'a pas encore. Si on ne peut pas s'appuyer sur des textes qui nous portent en avant, on n'avancera pas. C'est ce que j'aborde dans *Arbre de pertinence et utopie ". Car le symbole vient toujours couvrir la réalité. Et la mythologie a été le premier grand code symbolique.

V. \& I.: Vous voyez le symbole comme une main tranchante, mais n'êtes-vous pas tentée de créer des symboles vous aussi?

L. B.: Oui, d'une certaine façon, mais je veux aussi déjouer les symboles tráditionnels en littérature qui ont favorisé la coupure entre les sexes et installé entre eux la hiérarchie jusque dans la notion de genre dans la langue. Moi, je n'écris pas que les femmes sont la terre ou la lune. L'Euguélionne est une figure protéiforme, elle n'est jamais fixée. Je voulais qu'on ait au moins six versions de ce personnage quand elle arrive sur terre. Elle tourne sur elle-même et apparaît différente à chaque tour: elle est filiforme, puis grosse, elle est noire, puis c'est une rivière, puis c'est la fille d'une déesse australienne, etc. Je ne suis pas portée à fixer mes personnages, parce que ce fait le nie comme personnage, le fait mourir.

V. \& I.: Plus des figures que des symboles donc...

L. B.: Oui, des figures dynamiques comme la spirale, un peu comme je parle de la toile de l'araignée, figure dynamique, toujours en mouvement. L'Euguélionne pour moi est aussi une figure dynamique, un personnage qui me suscite. Elle ne transporte pas une doctrine, mais dit: Transgressez mes propres paroles. Voilà la clef de l'Euguélionne. Elle ne vient pas laisser un enseignement qu'il faudrait suivre. Je pense qu'il s'agit du plus grand message qu'on puisse 
apporter. Ce que je dis, faites-en votre miel, faites-en ce que vous pouvez en faire et continuez.

V. \& I.: N'est-ce pas d'ailleurs ce que vous avez fait vous-même avec les influences que vous avez eues?

L. B.: Oui. C'est ce que j'ai fait avec la modernité, avec l'Oulipo, avec la culture mass-médiatique. Quand j'écrivais l'Euguélionne, je prenais continuellement des notes, en écoutant la radio, les conversations autour de moi, en regardant la télévision, en lisant les journaux, je faisais mon miel avec tout ce qui me tombait sous la main.

V. \& I.: Vous vous référez constamment à l'Euguélionne. Est-ce le livre le plus important pour vous?

L. B.: C'est un texte générateur, c'est un texte de référence. Souvent quand je parle avec des amis, je dis: J'ai écrit ça dans l'Euguélionne. Il s'agit d'un personnage qui me sort de moi-même. Je suis timide, ramassée, effrayée, elle est fonceuse. Elle est immense. Comme les cariatides, elle a au moins neuf pieds de haut.

V. \& I.: Ny a-t-il pas un lien avec ce que vous appelez l'archéologie du futur?

L. B.: Quand j'affirme que j'écris pour faire l'archéologie du futur, c'est pour que la mémoire du futur s'inscrive dans le présent, de façon à ce que ce présent devienne une chose ancienne et dépassée. C'est la science fictive des monuments antiques de l'avenir. La mémoire du futur, c'est le fait d'imaginer un avenir qui ne serait pas entièrement sécrété par le passé. Cela contribue à me représenter cet avenir et, par conséquent, cela m'aide à vivre. Cette archéologie se fait dans le présent. Elle comporte tout ce qui est patriarcal actuellement et qui sera dépassé dans le futur.

V. \& I.: On en revient à l'utopie...

L. B.: Oui, mais l'u-topie n'est qu'une apparence. C'est un projet qui paraît irréalisable, mais qui ne l'est pas réellement. Je suis certaine que dans les temps les plus reculés, le patriarcat était de l'ordre de l'utopie dans l'imaginaire des hommes. Dans la mesure où notre réalité est couverte par le système symbolique patriarcal, on ne peut pas dire que l'utopie de la gynilité ne tient pas compte de la réalité. Elle est tout simplement un non-lieu provisoire. L'utopie, c'est donc ce que je peux faire, moi, après être passée à travers la mémoire du futur, en m'inspirant du passé, des figures mythologiques du passé. Toutes les figures que je peux créer et mettre dans mon arbre de pertinence. Comme si, à un moment donné, les femmes avaient pris leur place sur cette terre, non seulement une place concrète, mais une place dans l'imaginaire et le symbolique. On n'aurait plus besoin du féminisme, on serait rendues dans l'utopie de la gynilité. 
V. \& I.: Même si vous abordez des sujets extrêmement sérieux, votre écriture est souvent teintée d'humour...

L. B.: Je veux faire ressortir les étoiles qui brillent à travers les nuages. Il y a toujours un côté drôle dans un drame, ce n'est pas un hasard si on retrouve souvent des comédies dramatiques. Je ne voudrais pas que notre condition de femme soit un drame, parce que cela deviendrait du fatalisme. Il nous faut faire comme les petites souris du film la Cérémonie secrète. Deux souris tombent dans le lait. La première est tellement désespérée qu'elle se laisse aller et se noie. L'autre se débat tellement qu'elle baratte le lait qui devient du beurre, elle est sauvée. Je me retrouve là: je me débats dans l'existence pour ne pas couler. Il n'existe pas de moyen terme pour nous, les femmes: ou bien on se noie, ou bien on se dissout dans ses propres larmes, ou bien on devient des êtres utopiques. Je suis encore à la recherche de mon identité. J'arriverai peut-être un jour à écrire je. Je ne sais pas encore qui je suis, mais je sais en toute certitude qui je ne suis pas.

V. \& I.: Serait-il possible pour vous de séparer la question de l'engagement de celle de l'écriture?

L. B.: Pour moi, la question de l'engagement est imbriquée dans la question de l'écriture. Je ne peux écrire sans une conscience féministe, peu importe ce que j'écris, même de la poésie. On ne peut mettre sa conscience de côté quand on écrit. On est toujours engagé dans sa propre conscience. 